\title{
Integration Strategy for Standalone Compliant Interactive Systems for Add-on IoT based Electronics
}

\author{
Sherjeel Khan \\ mmh labs \\ King Abdullah University of Science and Technology \\ Thuwal, Saudi Arabia \\ ORCID ID: 0000-0001-6730-4330
}

\begin{abstract}
I present the idea and inspiration behind the selection of my Ph.D. topic and elaborate the work done until now with a brief look at the future work. My aim is to enhance the functionality of humans, plants, and things by providing them with add-on low-cost standalone flexible systems capable of integration with IoT based systems to make everything smart. For each subcategory, I chose a research problem to potentially find solutions. For humans, I am working on a foil based acoustic sensor optimized to monitor wheezing by designing its diaphragm. Match filtering will then be used to differentiate between different wheezing sounds. For plants, I am developing a standalone flexible ultra-lightweight platform that monitors the microclimate along plants more accurately from the leaf instead of the soil. For things, I plan to install paper-based humidity and temperature sensors to monitor pill health. The challenge is to figure out an integration strategy for incorporating paper sensors in electronic systems as paper sensors are incompatible with soldering.
\end{abstract}

Keywords—add-on; compliant; flexible; lightweight

\section{INTRODUCTION}

Physically compliant electronics are scientifically intriguing, mechanically complex, technologically challenging but socio-economically hugely potential. Until now the target applications for flexible electronics has been limited to display, solar cells, printed batteries, wearables and implantable for humans. However, with the emergence and growth of the Internet of Things (IoT) devices worldwide from nearly 27 billion in 2017 to 125 billion in 2030, in this Ph.D. research, expanding the horizon of applications for flexible electronics toward existing "things" will be explored. Using a low-cost fashion through the introduction of low-cost sustainable materials as active electronic materials and Do-It-Yourself (DIY) integration strategy to build "Add-on" standalone sensory system which can be attached to any existing things like a decal. Last but not least, they can also be tagged on living beings including plants. The core of the system will have flexible silicon CMOS ICs for data management, instead of conventional rigid ICs.

In my quest to present add-on systems, I will investigate, develop and optimize an integration strategy to build standalone compliant interactive system which can be used as add-on electronics to "smart" both things and living beings. This quest will involve the design of the standalone system, sustainable but unorthodox electronic material exploration, and a Do-itYourself (DIY) based integration strategy development.

\author{
Muhammad Mustafa Hussain \\ mmh labs \\ King Abdullah University of Science and Technology \\ Thuwal, Saudi Arabia \\ ORCID ID: 0000-0003-3279-044
}

\section{THE NEED FOR STANDALONE ADD-ON ELECTRONICS}

\section{A. Why Standalone Add-on Electronics?}

The importance of add-on can be explained best by using an example that would reach out to everyone. An important advantage of using an add-on approach is that we have so many things around us that have been used since ages but lack any sort of smart functionalities. It is difficult to reform the whole infrastructure as these things are deeply embedded in our ecosystems. One example could be the prescription bottles being used to give medicines to everyone. Currently, the world, especially the US, is facing a grave issue of prescription pill overuse. Using add-on the approach we can just replace the cap of the bottle with a smart cap laden with sensors and electronics that can keep track of the pill intake of patients. This work is disused in Section 5.

Add on presents another benefit where living beings such as humans and plants cannot be replaced with upgraded modules. For living beings like plants and humans, my objective is to develop a smart noninvasive module that can serve a specific purpose. In agriculture, technology can help improve the output of their crops is to understand how the temperature and humidity conditions affect the growth of plants. The challenge is to measure the ambient conditions around the plant leaves, where the critical photosynthesis process takes place, and monitor the growth of plants simultaneously. There is no reliable way to monitor the growth of plants where most researchers rely on looking video footage of a plant over time and estimate its growth. On the other hand, the sensors to monitor ambient conditions have to be placed in the soil as they are bulky. Thus they do not provide the accurate conditions experienced by the plant as the conditions can differ from the soil to the top of the plant. I present in Section 4, a smart wearable for plants that is extremely lightweight and can rest of the leaves of a plant to monitor the ambient conditions around the plant. Similarly, a lightweight growth monitor can be attached to the stem of the plant to monitor its growth quantitatively in real time. Similarly, humans also present a need for add-on electronics that are noninvasive and bio-friendly. For this purpose, I present in Chapter 3 one such application where a Styrofoam packaged paper sensor based sensor can be used to monitor respiratory conditions. The sensors are low cost, lightweight and made from recyclable materials in line with my vision of breaking the wall of expensive nonenvironmental friendly sensory systems. 


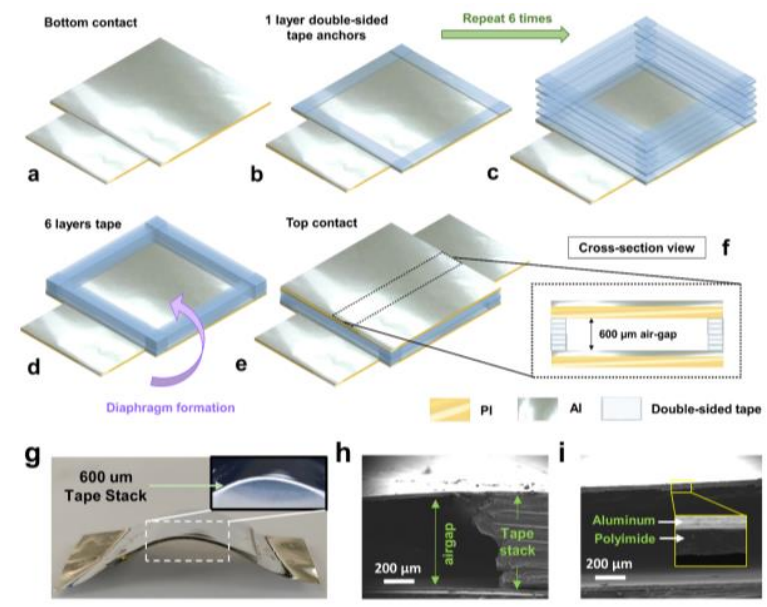

Fig. 1 (a) DIY Process flow of sensor fabrication. (b) Fabricated Sensor. SEM image at (c) edge showing tape stack (d) center showing air gap and Al-PI film stack

\section{B. Why Minimalistic DIY Approach?}

Another important aspect of my study is to fabricate these add-on electronics using DIY methods. One approach was to incorporate paper sensors which in addition to being low cost and recyclable, are compatible with DIY approaches. A simple scissor is used to cut sensors, and adhesive tapes are being used to bond them together while conductive copper/aluminum tapes are used to form electrical interconnects between sensors and electronics. Traditionally semiconductor-based IC package sensors require complex fabrication processes. They need foundries that cost millions of dollar to make sensors that are expensive, and no one else can make their customized versions. With my approach, we can customized version of sensors catered to our special needs. In addition, all the materials I use are readily available from the market at a low cost.

The sensors are not the only issue after the sensors one still needs to connect it to processors in order to process the data ta and take actions accordingly. This again requires a complex expensive process like soldering and printed circuit boards (PCB) manufacturing. Paper sensors are not compatible with such methods thus I had to form an integration strategy for such paper-based sensors. For that, I used anisotropic conductive tape to connect paper sensors with underlying electrical interconnects. Section 5 will elaborate on this strategy and show the mechanical contact studies that have been undergone to show the feasibility of it for use with paper-based sensors.

\section{ADD-ON FOR HUMANS}

In this section, I present a noninvasive add-on acoustic sensor made using paper-based products. By using recyclable paper-based materials, the complete system will be low cost and being noninvasive corroborates with my objective of enhancing the functionality of things using interactive add-on compliant systems created using DIY methods

\section{A. Fabrication flow}

The fabrication process is shown in Fig. 1. I began with a flexible conductive sheet as the bottom contact (Fig. 1a). To create the air-gap suspended design of our diaphragm, I used

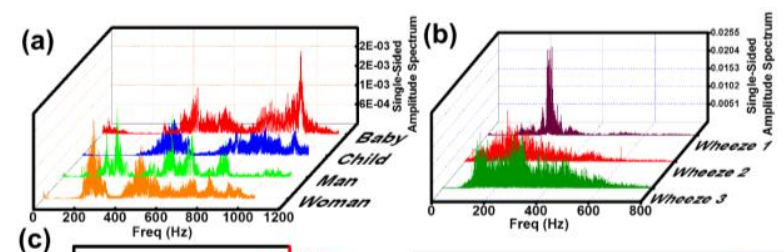

(c)
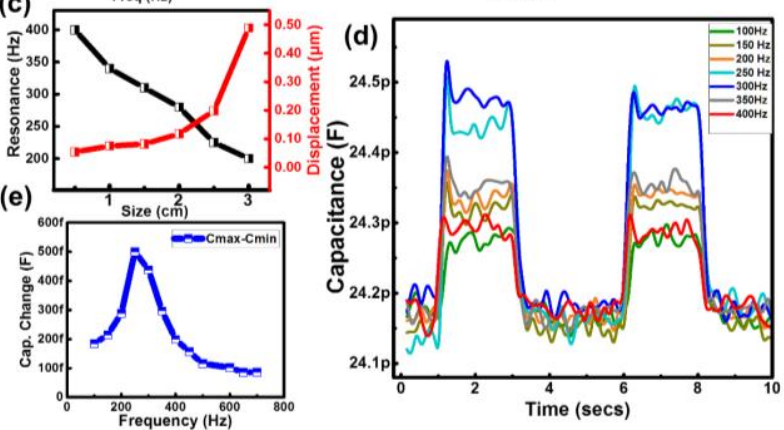

Fig. 2 (a) Frequency spectrum of sounds from talking of baby, child, man, and woman (b) Frequency spectrum of wheezing sounds (c) Resonance frequency vs displacement changes with diaphragm size variation (d) Frequency response of $2 \mathrm{~cm}$ sensor (e) capacitance change vs. frequency

six layers of double-sided adhesive tape strips (each strip was $1.2 \mathrm{~mm}$ wide and $\sim 90 \mu \mathrm{m}$ thick) as anchors along the four edges of the bottom contact (Fig. 1b) to create a gap of $>550 \mu \mathrm{m}$; thus, the top and bottom conductive sheets do not touch each other in the center of the diaphragm (Figs. 1c and 1d). The final air gap (including the adhesive) was $600 \mu \mathrm{m}$. Finally, I completed the structure with another 'flexible conductive sheet as the top contact (Fig. 1e). Fig. 1f shows a cross-section schematic of our acoustic sensor, so the wing the diaphragm design. The fabricated sensor is shown in the bent form in Fig. 1g; the inset highlights the small air gap between the two sheets. In order to verify the integrity of the structure, Scanning Electron Microscopy images of the sensor were taken at the center and the edge, as shown in Figs. $1 \mathrm{~h}$ and 1i, respectively. The height was $626 \mu \mathrm{m}$ at the edges and $500 \mu \mathrm{m}$ in the middle due to the effect of gravity on the center of the diaphragm.

\section{B. Diaphragm Design}

American Thoracic Society (ATS) defines wheezing as high-pitched continuous sounds with a dominant frequency of $\geq 400 \mathrm{~Hz}$ and length of $>250 \mathrm{~ms}$. Wheezing sounds need to pass through chest wall and skin, which absorbs higher frequencies resulting in wheezing sounds lying in the frequencies between $100-1200 \mathrm{~Hz}$. Our goal is to design a diaphragm such that it resonates at a frequency in between this range to obtain the maximum spectrum of wheezing and have maximum displacement corresponding to a larger change in the output signal (capacitance) form the sensor. Fast Fourier Transformation (FFT) is a popular method used for feature extraction from sounds. I took a sample of sounds by placing a microphone on the chest of 4 subjects; a baby, a boy, a man, and a woman, and analyzed their FFT spectrum in MATLAB shown in Fig. 2a. I then took three samples of wheezing sounds from an online database and observed their FFT shown in Fig. 2b. These show that most frequency components of wheezing sounds taken from chest lie between $100-400 \mathrm{~Hz}$ while talking sounds lie in $>400 \mathrm{~Hz}$ range. I want to design the sensor so that it resonates at a frequency $<400 \mathrm{~Hz}$ to maximize wheezing signal 

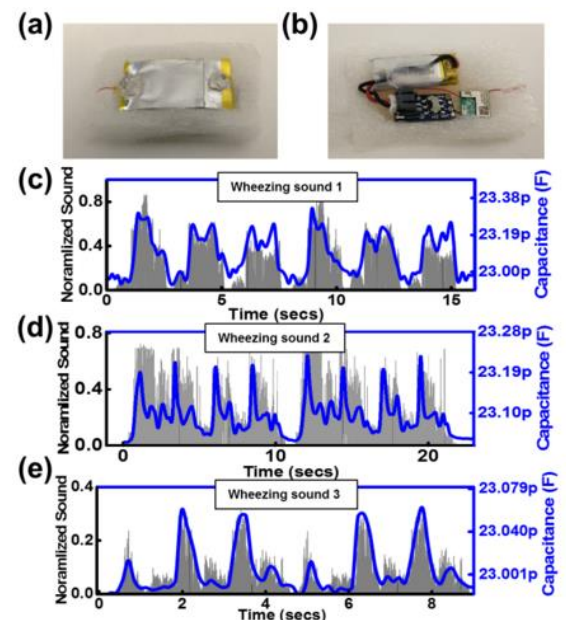

Fig. 3 (a) Sensor in Styrofoam package to reduce background noise (b) Wireless PSoC system on backside. Response of sensor (blue) vs original sound (grey) for wheezing sound (a) 1, (b) 2 (c) 3.a)

and reduce noise from talking. To find the desired size, I plotted both the response of deflection and frequency with varying sizes of diaphragm in-between $0.5-3.0 \mathrm{~cm}$ shown in Fig. 2c. The size on $\mathrm{x}$-axis depicts the length of each side of the square-shaped diaphragm. The deflection was calculated using FEM simulation of modeled Al-PI diaphragm, while resonance frequency was found experimentally by finding the response of sensor for sounds in between $100-400 \mathrm{~Hz}$. Resulting change in capacitance was plotted for each frequency, and the frequency of the maximum amplitude was taken as the resonance frequency. Fig. $2 \mathrm{~d}$ shows frequency response for a square diaphragm with $2 \mathrm{~cm}$ sides. I see that the maximum capacitance change, which corresponds to maximum deflection, occurs at $250 \mathrm{~Hz}$ as seen in Fig. 2e. Fig. 2c showed that sensor with a length of sides in between $1-2 \mathrm{~cm}$ has a resonance frequency in the desired range. However, smaller size has lesser deflection, and to get maximum output signal I select a $2 \mathrm{~cm}$ size for the sensor. The sensor seen in Figs. 3a-b was placed on the chest of a subject housed in Styrofoam packaging to reduce the effect of background noise. A Bluetooth speaker played sounds at a $2 \mathrm{~mm}$ distance from the sensor to mimic the gap later used in human trails. Figs. 3c-e show the signal from our sensor (data in blue) vs the signal plot of original wheezing sounds (grey data). Our sensor successfully detects sufficient features and differentiates between different wheezing sounds. For that, the matched filter will be used to detect wheezing sounds among the sound samples taken from the human chest.

\section{ADD-ON FOR PLANTS}

In this section, I present a flexible and stretchable "add-on" system to quantitatively monitor the health and growth of plants through the integration of temperature, humidity and strain sensors. The system will be extremely lightweight and noninvasive such that it can be placed on top of a mere leaf without affecting its function and monitor the environmental conditions around the plant to understand and improve plant growth..

\section{A. The need}

For the strain sensor fabrication, the goal is to build a stretchable and relatively long sensor that can be anchored from

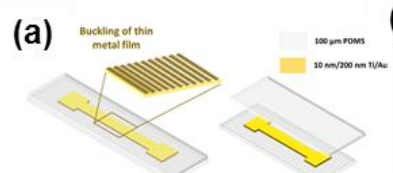

(b)

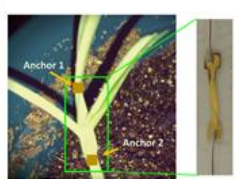

Fig. 4 - (a) Schematic of the strain sensor's process flow, highlighting the buckled thin metal film structure. (b) Digital photo of a Barley plant, showing the necessary placement of the strain sensor in order to monitor elongation and growth: anchor 1 refers to placement of contact pad 1 of the sensor, and anchor 2 refers to the position where the second contact pad of the sensor will be placed

one part on the bottom of the plant's stem, and the second anchor on one of the freshly grown leaves, as shown in Fig. 4b. The second challenging task was to make the sensor light in weight, enough not to bend the leaves or branches of the plant and inducing any unwanted fractures. The sensor of choice needs to be stretchable yet highly sensitive to strain, thus the design of choice was to use an intrinsically stretchable substrate material, Polydimethylsiloxane (PDMS), on top of which I deposit metal lines that act as the strain sensor (Fig. 4a). Since metals have a high Young's modulus, the metal would normally break after a couple of \% of stretching, leading a discontinuity in the grains of the metal film. Thus, to overcome this challenge, I implemented the buckling technique, which consists of prestraining the elastomer, then depositing the metal. After strain release, the substrate will go back to its original position and the metal film will compress to a smaller length by forming buckles (Fig. 4a). These buckles will allow the metal film to see less strain and withstand higher stretching before total discontinuity.

\section{ADD-ON FOR THINGS}

In this section, I present the plan and work done on a smart lid loaded with compliant paper-based sensors to record the temperature and humidity inside the bottle for pill health monitoring. I investigate an integration strategy of connecting paper sensors (which are incompatible with soldering and other electronic board manufacturing techniques) to existing electronics systems using a modular approach.

\section{A. Integration Strategy for paper-based sensors}

To make it compatible with a DIY assembly, I use an ACTbased sensor assembly approach, to integrate the sensor with electronic interfaces. For the same reason, I opt for a $3 \mathrm{M}^{\mathrm{TM}}$ Electrically Conductive Adhesive Transfer Tape 9703 consisting of a $50 \mu \mathrm{m}$ acrylic adhesive with silver particles spread across, at a pitch of $400 \mu \mathrm{m}$. When placed between two conductors and with the application of finger pressure, it forms an electrical contact between the top and bottom electrodes. As shown in Fig. 5a, when ACT is merely placed in-between two conductors, these conductors do not form an electrical contact as they are not in contact with the embedded silver particles. On application of finger pressure, the two conductors are pressed towards each other, inside the adhesive, and at some point, form an electrical contact after touching the silver particles from both sides. The acrylic adhesive binds the two conductors in the same place and this allows a current to flow anisotropically from one conductor to another. In order to study the electrical behavior and performance of the tape, I take a microscopic $\left(7 \mathrm{~mm}^{2}\right.$ area) image of the ACT (Fig. 5b). In order to count the number of 




h.

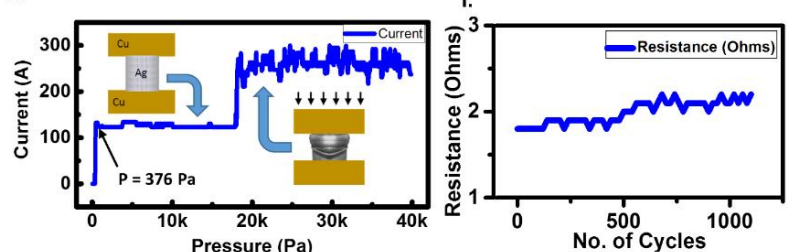

Fig. 5 (a) Illustration of how the anisotropic conductive tape (ACT) conducts current between two conductors, through embedded silver particles, after application of pressure. (b) Microscopic image of $7 \mathrm{~mm} 2$ area of the ACT with silver particles shown in dark color; transparent adhesive is shown in light color. (c) Processed image, using ImageJ software, to count the number of particles in the sample and their surface areas. (d) Histogram of distribution of silver particles according to their surface area. (e) FEM simulation of the replica model of $7 \mathrm{~mm} 2$ sample showing the electric current passing through the areas where silver particles are modeled. (f) FEM simulation of contact mechanics showing that pressures below $375 \mathrm{~Pa}$ do not form electrical contact through the prescribed $20 \mu \mathrm{m}$ gap between the ACT and the copper electrode. (g) FEM simulation showing contact is formed at $376 \mathrm{~Pa}$ pressure to overcome the $20 \mu \mathrm{m}$ gap between the ACT and copper. (h) Current passing through the modeled contact for pressures between $0-40 \mathrm{kPa}$. (i) Resistance change in the contact formed between the ACT and the copper tape, using finger pressure, for $11001 \mathrm{~cm}$ bending radius cycles.

particles in a given area and the average size of each particle, I process a sample of the ACT image, using Java based "ImageJ" software. First, the image must be converted to a binary image in order to be processed and for the software to perform the required calculations. After conversion and analysis by the image processing software, I can establish the number of particles in the sample and the particle's average surface area, as shown in Fig. 5c. From the data obtained, I plot the histogram of particle sizes spread across the sample (Fig. 5d). I obtain a value of $2000 \mu \mathrm{m} 2$ for the mean surface area of particles. Based on this information, I re-create a $7 \mathrm{~mm} 2$ model of the acrylic tape, with 360 silver cylindrical particles of $2000 \mu \mathrm{m} 2$ surface area and $50 \mu \mathrm{m}$ height, sandwiched between two copper electrodes. I then perform FEM electrical simulations with a potential of $5 \mathrm{~V}$ applied across the two copper conductors. The resulting current flow is presented in Fig. 5e. In this figure, I see that the areas containing silver particles conduct current. In an ideal case, the electrical contact becomes ohmic, with almost zero contact resistance. This happens when the two metals are in complete contact, due to the fact that the cylindrical silver (of $50 \mu \mathrm{m}$ height and $2000 \mu \mathrm{m}^{2}$ surface area) has a negligible resistance $(0.0403 \Omega)$ of its own. The value of current obtained from simulation results (118 A) corroborate the mathematical value of current (124 A) that flows when a $5 \mathrm{~V}$ potential is applied across a silver particle of $0.0403 \Omega$.

In a real-life non-ideal scenario, when two conductors are placed around the ACT, no electrical contact can be established since the pressure is required for the silver particles to form contact between the two conductors. To establish that pressure, I create a model of one silver particle (of $50 \mu \mathrm{m}$ height and $2000 \mu \mathrm{m} 2$ surface area) in between two conductors, with two conductors of $50 \times 100 \mu \mathrm{m} 2$ surface area (each) and a $50 \mu \mathrm{m}$ height. The size of silver particles is kept identical to that used in previous simulation studies, however, a $20 \mu \mathrm{m}$ gap is now created between the top conductor and the silver particle, with the goal of imitating the scenario when a conductor is merely placed on the ACT without any application of force. After performing FEM contact simulations in COMSOL, using this model, and by varying pressure and observing current conductivity, I find that electrical contact is established when a pressure of $376 \mathrm{~Pa}$ is applied. As shown in Fig. 5f, for a pressure of $375 \mathrm{~Pa}$ and below, no current flows through the silver particle and remains confined in the top electrode. At $376 \mathrm{~Pa}$, a current starts to flow between the two conductors through the silver particles in the ACT. From these experiments, we can conclude that a pressure of $376 \mathrm{~Pa}$ per silver particle (or per $5000 \mu \mathrm{m} 2$ area) is required to establish an electrical current. This corresponds to a pressure of $1.5 \mathrm{kPa} / \mathrm{cm} 2$ which can be easily applied by human finger [2]. However, at higher pressures, the shape of the particles will change (deform) and surface area between the particles will increase. This occurs because the silver particle, at the top of the cylinder, becomes compressed and forms an irregular shape. At this point, the conductance of the current increases. To study this behavior, I run a pressure sweep from 0 to $40 \mathrm{kPa}$, using on the same FEM model as I did for finding out the pressure needed to make a contact with a $20 \mu \mathrm{m}$ gap. I then observe the flow of the current at a fixed point on the silver particle, for different values of applied pressure (Fig. 5h). The plot is shown in Fig. 5h confirms the hypothesis that, after the first initial contact at $376 \mathrm{~Pa}$, the current remains almost constant until $20 \mathrm{kPa}$. However, for pressures above $20 \mathrm{kPa}$, the current suddenly increases suddenly and then oscillates. This behavior can be attributed to the deformation of the silver particle, which increases the contact area between the two electrodes. Since the material is under stress and the contact is not ideal, the current behavior is similar to a loose contact showing a current sparking phenomenon [3]. Since we are using inherently flexible materials to make our sensors, electrical connections, and the substrate, I further examine the performance of the electrical contact, using ACT under bending. I run the sample through 1100 cycles of $1 \mathrm{~cm}$ bending radius (this value represents the median bending radius of daily events that these systems can experience), using a custom-built bending machine; I then monitor the change in resistance over time. Fig. $5 \mathrm{i}$ shows the bending tests results in over 1100 cycles for which each resistance measurement is taken after every 20 cycles. The graph displayed in Fig. 5i shows the resistance to be $2.1+0.1 \Omega$ at the end of 1100 bending cycles.

\section{REFERENCES}

[1] C.-H. Lee, L.-W. Tang, and S. R. Popuri, "A study on the recycling of scrap integrated circuits by leaching," Waste Management \& Research, vol. 29, no. 7, pp. 677-685, 2011.

[2] A. D. Astin, "Finger force capability: measurement and prediction using anthropometric and myoelectric measures," Virginia Tech, 1999.

[3] J. Sletbak, R. Kristensen, H. Sundklakk, G. Navik, and M. Runde, "Glowing contact areas in loose copper wire connections," IEEE transactions on components, hybrids, and manufacturing technology, vol. 15 , no. 3, pp. 322-327, 1992. 